\title{
Optical constants of graphene measured by spectroscopic ellipsometry
}

\author{
J. W. Weber, ${ }^{1, a)}$ V. E. Calado, ${ }^{2}$ and M. C. M. van de Sanden ${ }^{1, b)}$ \\ ${ }^{1}$ Department of Applied Physics, Eindhoven University of Technology, P.O. Box 513, 5600 MB Eindhoven, \\ The Netherlands \\ ${ }^{2}$ Department of Applied Physics, Delft University of Technology, Lorentzweg 1, 2628 CJ Delft, \\ The Netherlands
}

(Received 12 April 2010; accepted 13 July 2010; published online 31 August 2010)

\begin{abstract}
A mechanically exfoliated graphene flake $\left(\sim 150 \times 380 \mu \mathrm{m}^{2}\right)$ on a silicon wafer with $98 \mathrm{~nm}$ silicon dioxide on top was scanned with a spectroscopic ellipsometer with a focused spot $(\sim 100$ $\times 55 \mu^{2}$ ) at an angle of $55^{\circ}$. The spectroscopic ellipsometric data were analyzed with an optical model in which the optical constants were parameterized by B-splines. This parameterization is the key for the simultaneous accurate determination of the optical constants in the wavelength range 210-1000 nm and the thickness of graphene, which was found to be $3.4 \AA$. () 2010 American Institute of Physics. [doi:10.1063/1.3475393]
\end{abstract}

In 2004, it was discovered that a free-standing single atomic layer can be isolated from its environment by means of micromechanical cleavage. ${ }^{1}$ Of the different reported twodimensional crystals, the single atomic layer of graphite, graphene, has gained most interesting due to its remarkable electronic properties. ${ }^{2}$ The vast majority of the studies focuses therefore on its electronic properties. Its optical properties, however, were less explored.

Gray et $\mathrm{al}^{3}{ }^{3}$ studied the optical properties of graphene by near-normal incidence reflectance measurements in the range 190-1000 nm. They acquired reflectance data of graphite flakes of different thicknesses, down to graphene, deposited on a silicon wafer with $300 \mathrm{~nm}$ silicon dioxide $\left(\mathrm{SiO}_{2}\right)$ on top. They assumed the optical constants to be independent of thickness and that they could be parameterized with five Forouhi-Bloomer oscillators. The parameters of these oscillators and each thickness were fitted simultaneously to all the reflectance data. The thickness was fitted as $3.8 \AA$. This work was extended by adding spectroscopic ellipsometry and $s$-polarized reflectance (both at $70^{\circ}, 380-1000 \mathrm{~nm}$ ) to the near-normal incidence reflectance in their data analysis. ${ }^{4}$ This time, however, the optical constants were not assumed to be independent of thickness and were parameterized by a proprietary dispersion model. The dispersion parameters, however, were not reported. They found the thickness of graphene was $3.7 \AA$. Very recently Kravets et al..$^{5}$ also used spectroscopic ellipsometry on graphene on an oxidized silicon wafer (300 nm $\mathrm{SiO}_{2}$ ), and on amorphous quartz. They report optical constants extracted from the variable angle $\left(45^{\circ}-70^{\circ}\right)$ ellipsometry data by numerical inversion in the range $240-750 \mathrm{~nm}$ for the amorphous quartz wafer (240$1000 \mathrm{~nm}$ for the oxidized wafer), assuming a thickness of $3.35 \AA$.

In this paper we show the optical constants and report dispersion parameters of graphene as found from spectroscopic ellipsometry in the range 210-1000 nm. We show that, without assuming any physical oscillator parameterization beforehand, B-splines allow an uncorrelated, accurate, and simultaneous determination of the optical constants and

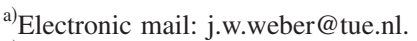

${ }^{b)}$ Electronic mail: m.c.m.v.d.sanden@tue.nl.
}

thickness of graphene. The thickness is in perfect agreement with the thickness as expected from the interlayer spacing in graphite: $3.4 \AA$. Based on the found optical constants we have simulated transmittance for graphene. We show that this simulation is in better agreement with measured transmittance ${ }^{6}$ than the transmittance that we simulated based on optical constants from other work. ${ }^{3,7}$

Ellipsometry can measure the change in polarization of light after reflection from a sample. ${ }^{8}$ This change is measured as the ratio of the Fresnel reflection coefficients for the $p$ and $s$ component of the reflected light, denoted as $r_{p}$ and $r_{s}$, respectively. This ratio, $\rho$, is in general a complex number and commonly expressed as $\rho=r_{p} / r_{s}=\tan \Psi \exp \mathrm{i} \Delta$, where $\Psi$ and $\Delta$ are the ellipsometric angles.

We used an automated angle M-2000F rotating compensator ellipsometer with a $300 \mathrm{~mm} \mathrm{X}$-Y mapping stage and focusing probes, and the accompanying software CompleteEASE 4.27 from J.A. Woollam Co., Inc. A rotating compensator ellipsometer can measure all of the four Stokes parameters, $S_{0}$ to $S_{3}$, in a single measurement. ${ }^{9}$ The degree of depolarization, defined as $p=\left(S_{1}^{2}+S_{2}^{2}+S_{3}^{2}\right)^{1 / 2} / S_{0}$, for the measurements on graphene is on average $1.3 \%$. The ellipsometric data were acquired in the wavelength range $\lambda$ $=210-1000 \mathrm{~nm}$ with a resolution of $\Delta \lambda \simeq 1.6 \mathrm{~nm}$ at an angle of incidence $\theta=55^{\circ}$. At this angle the spot size is small enough to acquire several scans from the graphene flake. The acquisition time per measurement is $1 \mathrm{~min}$, resulting in a very high signal to noise ratio. Our single layer graphene was prepared by mechanical cleavage of natural graphite (NGS Naturgraphit GmbH). ${ }^{2}$ Raman measurements at $514 \mathrm{~nm}$, confirmed that it is a monolayer: the intense 2D-peak at $\sim 2690 \mathrm{~cm}^{-1}$ and the intense $G$-peak at $\sim 1580 \mathrm{~cm}^{-1}$ correspond to the peaks for graphene. ${ }^{10}$

To extract optical constants and thickness of a sample from ellipsometric data, an optical model is required that describes the sample's optical response. It consists of the thickness and (parameterizations for) the optical constants of every layer in the sample. The "goodness-of-fit" of the model to the experimental data is determined by the reduced chisquared unbiased estimator, $\chi_{\text {red }}^{2}$, for the three Stokes parameters $S_{1}$ to $S_{3}$ 

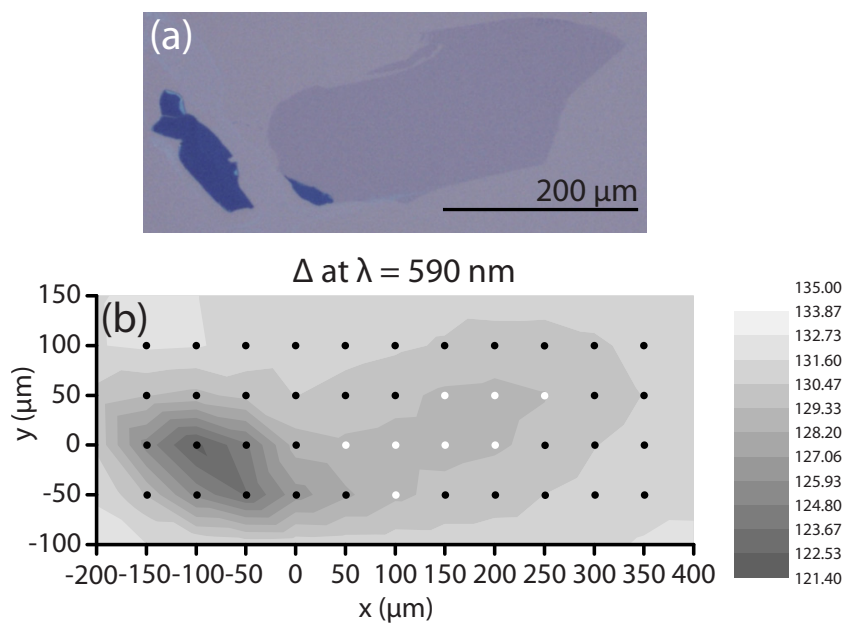

FIG. 1. (Color online) (a) An optical microscope image of graphene exposed to visible light. The darker part is multilayer graphene. (b) A spectroscopic ellipsometric scan of the flake showing a map of $\Delta$ at $590 \mathrm{~nm}$. The white dots indicate the positions from which the spectra can be attributed to originate from the graphene only. The shape that encloses the white spots is similar to that of the flake.

$$
\chi_{\mathrm{red}}^{2}=\frac{1}{3 n-m} \sum_{j=1}^{n} \sum_{i=1}^{3}\left(\frac{S_{i, j}^{\exp }-S_{i, j}^{\mathrm{mod}}}{\sigma_{S_{i, j}}^{\exp }}\right)^{2},
$$

where $n$ is the number of wavelengths, $m$ is the number of fit parameters and $\sigma_{S}$ is the error in the determined Stokes parameter. This error is assumed to be equal for all three parameters and for all wavelengths: $\sigma_{\mathrm{S}}=0.001{ }^{11}$ While fitting, $\chi_{\text {red }}^{2}$ is minimized by the Levenberg-Marquardt nonlinear regression algorithm.

We study a three layer structure consisting of a single side polished crystalline silicon (c-Si) substrate, a $\mathrm{SiO}_{2}$ layer, and graphene. To determine the optical constants and thickness of graphene as accurately as possible, it is crucial that the optical response of the underlying layers is well known. We therefore acquire data of four spots next to the graphene flake. Since the optical constants of $\mathrm{c}-\mathrm{Si}$ are well known from literature ${ }^{12}$ and the substrate can be considered to have a semi-infinite thickness, it is only necessary to find the $\mathrm{SiO}_{2}$ thickness and optical constants, which we first parameterize with a Sellmeier dispersion relation. ${ }^{11}$ For the four measurements simultaneously, the three Sellmeier parameters and thickness are fitted together with an offset for the angle of incidence. A unique solution is then found, with $\chi_{\text {red }} \simeq 3.61$. To match the experimental data and the model the closest possible, the thickness, and angle offset found are fixed and $\Psi$ and $\Delta$ are then numerically inverted ${ }^{9}$ to $n$ and $k$ with $\chi_{\text {red }} \simeq 0.827$. These optical constants together with the thickness and angle offset from the Sellmeier dispersion fit, are used to characterize the $\mathrm{SiO}_{2}$ layer.

The $\mathrm{SiO}_{2}$ layer serves to increase the contrast due to interference enhancement. In this study the thickness of the $\mathrm{SiO}_{2}$ layer is $98 \mathrm{~nm}$. For this $\mathrm{SiO}_{2}$ thickness the contrast window is broader than the commonly ${ }^{3,13}$ used $300 \mathrm{~nm} \mathrm{SiO}_{2}$ and allows the graphene flake to be detected easily under visible light. ${ }^{14}$ Due to the broader contrast also the sensitivity for the fitted optical constants increases and hence their accuracy. Figure 1(a) shows an optical microscope image of the graphene flake exposed to visible light.
Once the optical response of the underlying layers is characterized very accurately, the third layer for graphene is added for the analysis of the measurements on graphene. Since we want to determine the optical constants and thickness of graphene independently, we do not use numerical inversion, assuming a thickness. Instead, we use a parameterization for the optical constants. Since we do not want to assume any physical oscillator parameterization beforehand, we use a B-spline function, which is defined as a linear sum of B-splines

$$
S(x)=\sum_{i=1}^{n} c_{i} B_{i}^{k}(x),
$$

in which $c_{i}$ are the B-spline coefficients. B-splines are a special set of piecewise defined polynomials and can be given by the following recursive formula:

$$
\begin{aligned}
& B_{i}^{0}(x)=\left\{\begin{array}{cc}
1 & t_{i} \leq x \leq t_{i+1} \\
0 & \text { otherwise }
\end{array},\right. \\
& B_{i}^{k}(x)=\left(\frac{x-t_{i}}{t_{i+k}-t_{i}}\right) B_{i}^{k-1}(x)+\left(\frac{t_{i+k+1}-x}{t_{i+k+1}-t_{i+1}}\right) B_{i+1}^{k-1}(x),
\end{aligned}
$$

in which $t$ are the abscissa of the knots, which are the points where the polynomials connect, and $k$ is the B-spline degree. ${ }^{15}$ For $n$ knots there are $n-k-1$ coefficients; no coefficients exist for $t_{1}, \ldots t_{k-1}$ and $t_{n-k+2}, \ldots t_{n} .{ }^{16}$ The B-spline coefficients are the fit parameters whereas the knots are chosen. Since a Kramers-Kronig (KK) transformation exists for a B-spline function, ${ }^{15}$ we can enforce KK consistency on our optical constants during fitting. This not only ensures a physical solution but also reduces the number of fitting parameters by two, since now only $n$ needs to be found and $k$ can be found from the KK transformation (or vice versa). Our analysis software can only report coefficients for the imaginary part of the dielectric function, $\varepsilon_{2}$, as a function of energy: $\varepsilon_{2}(E)=\sum_{i=1}^{n} c_{i} B_{i}^{k}(E)$ (the real part of the dielectric function, $\varepsilon_{1}$, is found from the $\mathrm{KK}$ transformation). We therefore report $t_{i}$ in electronvolt and $c_{i}$ for $\varepsilon_{2}$. Since a B-spline function has ultimate shape control it can follow all the features in the optical function, depending on the amount of knots, while still being KK consistent.

To determine the thickness and optical constants of graphene, only these data should be analyzed that are acquired from the graphene flake and not also partially from the $\mathrm{SiO}_{2}$. The graphene flake is $\sim 150 \times 380 \mu \mathrm{m}^{2}$, and we measured the full width at half maximum spot size as $\sim 100$ $\times 55 \mu \mathrm{m}^{2}$ with a knife-edge type of technique. Since the scan step size is $50 \mu \mathrm{m}$, there should be several measurements only on the graphene flake, showing the same spectra. We identified eight spots of which the spectra overlapped. These eight spots are shown as the white dots in Fig. 1(b). The shape that encloses the white spots is similar to that of the picture of Fig. 1(a).

The optical model is fitted to these eight spectra simultaneously. The B-spline parameterization has a degree $k=3$. We chose ten knots, with a spacing of $0.5 \mathrm{eV}$ in the measured range, and three knots outside the measured range. One of these three knots is necessary for absorption in the infrared and the other two for absorption in the ultraviolet range. The outer four knots ensure that $\varepsilon_{2}$ goes smoothly to zero. A total of thirteen coefficients is fitted. This number of coefficients 
TABLE I. B-spline knots, $t_{i}$, and $\varepsilon_{2}$-coefficients, $c_{i}$.

\begin{tabular}{lccccc}
\hline \hline \multicolumn{1}{c}{$t_{i}$} & $c_{i}$ & $t_{i}$ & $c_{i}$ & $t_{i}$ & $c_{i}$ \\
\hline-0.4 & $\mathrm{n} / \mathrm{a}$ & 2.788 & 6.1277 & 5.880 & -0.0922 \\
-0.2 & $\mathrm{n} / \mathrm{a}$ & 3.303 & 6.1478 & 6.380 & 3.6559 \\
0 & 28.9227 & 3.818 & 5.6179 & 6.880 & 12.1830 \\
1.241 & 11.0713 & 4.334 & 21.3483 & 7.880 & $\mathrm{n} / \mathrm{a}$ \\
1.757 & 7.9615 & 4.849 & 4.2674 & 9.880 & $\mathrm{n} / \mathrm{a}$ \\
2.272 & 6.4513 & 5.365 & 2.8995 & & \\
\hline \hline
\end{tabular}

proved to be sufficient to achieve both a very good fit and still be small enough to avoid correlation between all the fit parameters. Together with fitting the thickness a $\chi_{\text {red }}=2.454$ is obtained, which means a very good fit considering that eight spectra are fitted simultaneously.

The thickness was fitted as $3.4 \pm 0.04 \AA$. To test its uniqueness, the thickness is changed over a range of values. At each value, the thickness fit parameter is fixed while all other fit parameters are varied to find the lowest $\chi_{\text {red. }}$ The uniqueness of the fitted thickness is shown in the inset of Fig. 2: a minimum for $\chi_{\text {red }}$ is at $3.4 \AA$. In Table I the B-spline knots and coefficients are shown. In Fig. 2 the optical constants, $n$ and $k$, of graphene are shown as functions of wavelength. An intense peak in $k$ is observed at $270 \mathrm{~nm}(4.6 \mathrm{eV})$. This peak can be attributed to the effect of strong resonant excitons. ${ }^{17}$ Compared to Kravets et al., ${ }^{5}$ the optical constants in Fig. 2 are smooth and KK consistent and the peak at 270 $\mathrm{nm}$ is even more intense.

Based on these optical constants we simulated the transmittance for freestanding graphene and compared it to the transmittance as measured by Nair et al., ${ }^{6}$ and as modeled from the optical constants found by Gray et al., ${ }^{3}$ and Bruna and Borini. The latter used the measured transmittance by Nair et ll. $^{6}$ and modeled the optical constants of graphene in the visible wavelength range by a constant refractive index and a linear dispersion for the extinction coefficient: $n=3$ and $k=\left(C_{1} / n\right) \lambda$, with $C_{1}=5.446 \mu \mathrm{m}^{-1}$. The comparison in Fig. 3 shows that the transmittance as modeled from the optical constants in this work agrees better with the measured transmittance than the transmittance as modeled from the optical constants by Gray et al. ${ }^{3}$ It also agrees better than the modeled transmittance of Bruna and Borini, ${ }^{7}$ especially towards higher energies where there is the onset of the absorption peak at $4.6 \mathrm{eV}$.

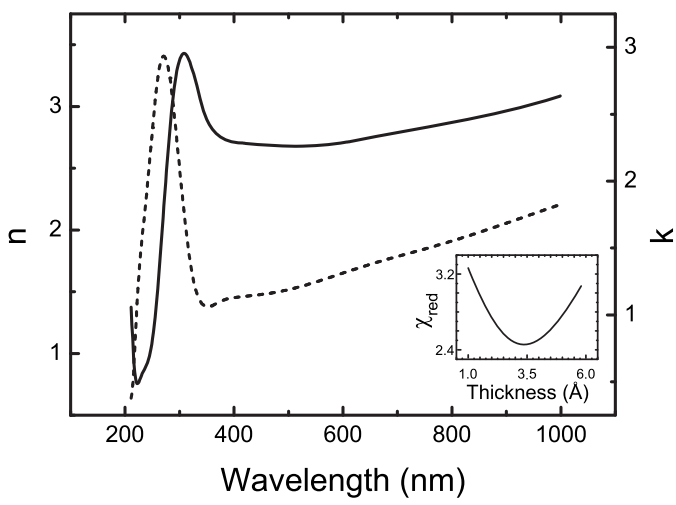

FIG. 2. Optical constants of graphene $n$ (solid line) and $k$ (dashed line). Inset: $\chi_{\text {red }}$ as a function of the thickness fit parameter; a unique minimum is found for $3.4 \AA$.

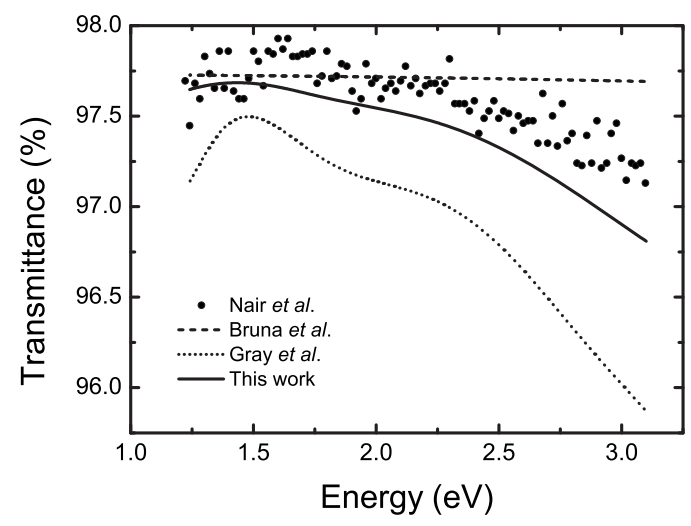

FIG. 3. Transmittance of graphene as measured by Nair et al. (Ref. 6), and as modeled from the optical constants as found by Bruna et al. (Ref. 7), Gray et al. (Ref. 3), and of this work.

In summary, spectroscopic ellipsometry in combination with a B-spline parameterization, allowed an accurate determination of the thickness of graphene and its KK consistent optical constants for the range $210-1000 \mathrm{~nm}$. The thickness was fitted as $3.4 \AA$, which is in perfect agreement with the interlayer spacing in graphite. Based on the optical constants we simulated transmittance for freestanding graphene in the visible range and found good agreement with measured transmittance.

We thank Maarten van Kampen and Goran Milinkovic. This work is part of the research programme of the Foundation for Fundamental Research on Matter (FOM), which is financially supported by the Netherlands Organisation for Scientific Research (NWO). The grant for this work is part of the FOM Valorization Prize 2009.

${ }^{1}$ K. S. Novoselov, D. Jiang, F. Schedin, T. J. Booth, V. V. Khotkevich, S. V. Morozov, and A. K. Geim, Proc. Natl. Acad. Sci. U.S.A. 102, 10451 (2005).

${ }^{2}$ K. S. Novoselov, A. K. Geim, S. V. Morozov, D. Jiang, Y. Zhang, S. V. Dubonos, I. V. Grigorieva, and A. A. Firsov, Science 306, 666 (2004).

${ }^{3}$ A. Gray, M. Balooch, S. Allegret, S. De Gendt, and W.-E. Wang, J. Appl. Phys. 104, 053109 (2008).

${ }^{4}$ W. E. Wang, M. Balooch, C. Claypool, M. Zawaideh, and K. Farnaam, Solid State Technol. 52 (6), 18 (2009).

${ }^{5}$ V. G. Kravets, A. N. Grigorenko, R. R. Nair, P. Blake, S. Anissimova, K. S. Novoselov, and A. K. Geim, Phys. Rev. B 81, 155413 (2010).

${ }^{6}$ R. R. Nair, P. Blake, A. N. Grigorenko, K. S. Novoselov, T. J. Booth, T. Stauber, N. M. R. Peres, and A. K. Geim, Science 320, 1308 (2008).

${ }^{7}$ M. Bruna and S. Borini, Appl. Phys. Lett. 94, 031901 (2009).

${ }^{8}$ Handbook of Ellipsometry, edited by H. G. Tompkins and E. A. Irene (William Andrew, Norwich, New York, 2005).

${ }^{9}$ H. Fujiwara, Spectroscopic Ellipsometry: Principles and Applications (Wiley, New York, 2007).

${ }^{10}$ A. C. Ferrari, J. C. Meyer, V. Scardaci, C. Casiraghi, M. Lazzeri, F. Mauri, S. Piscanec, D. Jiang, K. S. Novoselov, S. Roth, and A. K. Geim, Phys. Rev. Lett. 97, 187401 (2006)

${ }^{11}$ CompleteEASE ${ }^{\mathrm{TM}}$ Data Analysis Manual Version 4.05 (J.A. Woollam Co., Inc., Lincoln, NE, 2009).

${ }^{12}$ C. M. Herzinger, B. Johs, W. A. McGahan, J. A. Woollam, and W. Paulson, J. Appl. Phys. 83, 3323 (1998).

${ }^{13}$ A. K. Geim and K. S. Novoselov, Nature Mater. 6, 183 (2007).

${ }^{14}$ P. Blake, E. W. Hill, A. H. C. Neto, K. S. Novoselov, D. Jiang, R. Yang, T. J. Booth, and A. K. Geim, Appl. Phys. Lett. 91, 063124 (2007).

${ }^{15}$ B. Johs and J. S. Hale, Phys. Status Solidi A 205, 715 (2008).

${ }^{16} \mathrm{~J}$. W. Weber, T. A. R. Hansen, M. C. M. van de Sanden, and R. Engeln, J. Appl. Phys. 106, 123503 (2009).

${ }^{17}$ L. Yang, J. Deslippe, C.-H. Park, M. L. Cohen, and S. G. Louie, Phys. Rev. Lett. 103, 186802 (2009). 